\title{
ASSISTÊNCIA DE ENFERMAGEM NA SEGURANÇA DO PACIENTE CIRÚRGICO: REVISÃO INTEGRATIVA
}

\author{
Amanda Haissa Barros Henriques ${ }^{1}$, Suzana Santos da Costa ${ }^{2}$, Janice de Sousa Lacerda ${ }^{3}$
}

\begin{abstract}
RESUMO: O estudo objetivou analisar os achados científicos acerca da atuação do enfermeiro na promoção da segurança do paciente cirúrgico, identificar riscos e apontar soluções para a melhoria da assistência. É uma revisão integrativa realizada em três bases de dados da saúde nos meses de janeiro e fevereiro de 2016. Foram selecionados 28 artigos que se enquadraram nos critérios de inclusão estabelecidos, formando três categorias temáticas: Reflexão e Avaliação acerca da assistência ofertada ao paciente cirúrgico, Principais erros e fragilidades que põem em risco a segurança do paciente cirúrgico, e Instrumentos e Estratégias para favorecer a qualidade da assistência e segurança do paciente. Percebe-se que a temática da segurança do paciente necessita ser melhor discutida e posta em prática, visto que o desenvolvimento de mais estudos com essa abordagem pode contribuir para melhorias na assistência ao paciente cirúrgico.
\end{abstract}

DESCRITORES: Segurança do paciente; Enfermagem cirúrgica; Centro cirúrgico; Enfermagem perioperatória; Cuidados de enfermagem.

\section{NURSING CARE IN SURGICAL PATIENT SAFETY: AN INTEGRATIVE REVIEW}

ABSTRACT: The objective was to analyze the scientific findings on nurses' work in the promotion of surgical patient safety, to identify the risks and appoint solutions to improve care. An integrative review was undertaken in three health databases in January and February 2016 Twenty-eight articles were selected that complied with the inclusion criteria, in three thematic categories: Reflection and Assessment of the care offered to surgical patients, Main errors and weaknesses that put the safety of surgical patients at risk, and Tools and Strategies to favor the quality of patient care and safety. The theme patient safety needs to be discussed better and put in practice, as the development of further research with this approach can contribute to improvements in surgical patient care.

DESCRIPTORS: Patient safety; Surgical nursing; Surgicenter; Perioperative nursing; Nursing care.

\section{ATENCIÓN DE ENFERMERÍA EN LA SEGURIDAD DEL PACIENTE QUIRÚRGICO: UNA REVISIÓN INTEGRADORA}

RESUMEN: La finalidad del estudio fue analizar los hallazgos científicos acerca de la actuación del enfermero en la promoción de la seguridad del paciente quirúrgico, identificar riesgos e indicar soluciones para la mejora de la atención. Fue llevada a cabo una revisión integradora en tres bases de datos de la salud en los meses de enero y febrero del 2016. Fueron elegidos 28 artículos que cumplieron con los criterios de inclusión establecidos, constituyendo tres categorías temáticas: Reflexión y Evaluación acerca de la atención ofertada al paciente quirúrgico, Principales errores y fragilidades que ponen en riesgo la seguridad del paciente quirúrgico, e Instrumentos y Estrategias para favorecer la calidad de la atención y seguridad del paciente. Se percibe que el tema de la seguridad del paciente necesita ser mejor discutida y puesta en práctica, ya que el desarrollo de otros estudios con esa aproximación puede contribuir para mejoras en la atención al paciente quirúrgico.

DESCRIPTORES: Seguridad del paciente; Enfermería quirúrgica; Centro quirúrgico; Enfermería perioperatoria; Atención de enfermería.

${ }^{1}$ Enfermeira, Mestre em Enfermagem. Docente de Enfermagem do Instituto Federal de Educação, Ciência e Tecnologia do Pernambuco. Belo Jardim, PE, Brasil.

${ }^{2}$ Enfermeira, Mestre em Enfermagem. Docente de Enfermagem do Centro Universitário do Vale do Ipojuca. Caruaru, PE, Brasil. ${ }^{3}$ Enfermeira, Mestre em Enfermagem. Enfermeira Assistencial, Hospital Dep. Manoel Gonçalves de Abrantes. Sousa, PB, Brasil. 


\section{- INTRODUÇÃO}

Os avanços tecnológicos e científicos na área da saúde têm propiciado aumento significativo no número de intervenções cirúrgicas, as quais são, muitas vezes, realizadas em condições inseguras, interferindo na promoção e na recuperação da saúde dos pacientes. Assim, a insegurança e vulnerabilidade dos clientes tem-se ampliado consideravelmente no meio científico e assistencial, visto que a ocorrência de eventos adversos acentua-se nas instituições hospitalares, representando grave problema de saúde pública ${ }^{(1)}$.

Pela segurança do cliente, busca-se a redução e/ou atenuação de atos considerados inseguros atrelados ao sistema de assistência à saúde, bem como o emprego das melhores práticas, no intuito de obter os resultados esperados. Ressalta-se ainda que o cuidado seguro reflete direta e intimamente a qualidade da assistência prestada à população; esta, por sua vez, tem adquirido mais ciência de seus direitos, estabelecendo critérios que refletem suas escolhas e atitudes diante dos serviços de saúde, visando, assim, evitar a ocorrência de eventos adversos, torná-los visíveis se ocorrerem e minimizar seus efeitos com intervenções eficazes ${ }^{(1)}$.

No hospital, o Centro Cirúrgico (CC) é o local onde acontece grande parte dos eventos adversos à saúde dos pacientes. Sua causa é multifatorial e atribuída à complexidade dos procedimentos, à interação das equipes interdisciplinares e ao trabalho sob pressão, pois, apesar de as intervenções cirúrgicas integrarem a assistência à saúde, contribuindo para a prevenção de agravos à integridade física e à perda de vidas, estão associadas, consideravelmente, aos riscos de complicações e morte ${ }^{(2-3)}$.

Estima-se que, anualmente, das 234 milhões de cirurgias realizadas pelo mundo, ocorram 2 milhões de óbitos e 7 milhões de pacientes sofram complicações após a cirurgia, das quais 50\% poderiam ter sido evitados. Dentre as cirurgias de alta complexidade realizadas em países desenvolvidos, registram-se complicações em torno de 3 a 16\%, e, a cada 300 pacientes admitidos, ocorre 1 óbito ${ }^{(1,4)}$.

A Aliança Mundial para Segurança do Paciente, na segunda Campanha Mundial, a Cirurgia Segura Salva Vidas, teve como um dos objetivos reforçar as práticas de segurança cirúrgica estabelecidas pela Organização Mundial da Saúde $(\mathrm{OMS})^{(5)}$. O programa abordou importantes questões de segurança, como as inadequadas práticas de segurança anestésica, as infecções cirúrgicas que podem ser evitadas e a escassa comunicação entre os membros da equipe cirúrgica(6).

Entende-se que a atividade no bloco cirúrgico envolve tarefas complexas, plenas de variação e de incerteza, exercidas em condições ambientais dominadas pela agilidade, precisão e pelo estresse. Logo, essas atividades requerem do profissional atenção redobrada nos processos que envolvem o paciente, em especial em relação ao enfermeiro, pelo contato próximo em suas ações assistenciais ao cliente ${ }^{(7)}$.

É atribuição do enfermeiro, portanto, informar o paciente cirúrgico sobre seu problema de saúde, procedimento cirúrgico e, principalmente, como ele poderá participar de sua recuperação pós-operatória, orientando-o no pré e pós-operatório por meio de uma linguagem clara, respeitando seus conhecimentos e sua cultura ${ }^{(8)}$.

É inerente ao papel do enfermeiro a atividade educativa, pois ele permanece diuturnamente com o paciente, podendo promover espaço propício para a orientação e o empoderamento sobre seu autocuidado, fornecendo orientações acerca dos procedimentos e cuidados, além de promover a saúde e prevenir complicações potenciais. Essa situação torna esse profissional o elemento da equipe de saúde com maior oportunidade para orientar o paciente ${ }^{(8)}$.

Além do mais, é nítida a importância da contribuição da enfermagem no início, durante e após o término da cirurgia, por exemplo, certificando a identidade e o consentimento do cliente, o local e o procedimento; verificando os sinais vitais e mantendo o paciente monitorado e com acesso venoso; eliminando a presença de qualquer risco, como o de perda sanguínea, dificuldade das vias aéreas, reações alérgicas e complicações no pós-operatório; entre outras funções ${ }^{(1)}$.

Por outro lado, identificar situações que remetam a possíveis erros na assistência perioperatória ao paciente cirúrgico é o grande desafio do enfermeiro, dentre as quais se destacam as falhas nos equipamentos de anestesia, falta de pessoal capacitado, equipe cirúrgica trabalhando sob pressão, uso das novas tecnologias com pouco conhecimento, entre outras. Esse contexto desperta atenção para ampliar o olhar do enfermeiro em busca de novos conhecimentos, visando à segurança do paciente no centro cirúrgico e tendo em vista 
a constante ocorrência de riscos, erros e acidentes, revelando a necessidade de que mudanças sejam implementadas ${ }^{(9)}$.

Assim, a mudança científica, assistencial e cultural acerca da segurança do paciente cirúrgico é crucial para que sejam implantadas medidas eficientes de prevenção e redução dos riscos e eventos adversos. Mostra-se necessária a construção de uma cultura que entenda os valores, as crenças e as normas daquilo que é importante em uma instituição e quais atitudes e comportamentos relacionados à segurança do paciente são necessários, recompensados e esperados ${ }^{(10)}$.

Sendo assim, implementar a cultura de segurança nas instituições de saúde, especificamente no Centro Cirúrgico, pode ter associação direta com a diminuição dos eventos adversos e da mortalidade, implicando melhorias na qualidade da assistência à saúde dos pacientes.

Partindo-se desse pressuposto, das atualizações constantes no âmbito profissional da segurança do paciente e da preocupação da OMS com a cirurgia segura para os pacientes do Sistema Único de Saúde (SUS), espera-se favorecer ao paciente um processo de recuperação positivo, protegido de eventos adversos relacionados à assistência insegura e despreparada.

Dessa forma, o presente estudo teve por objetivo analisar os achados científicos atuais acerca da atuação do enfermeiro na promoção da segurança do paciente cirúrgico, visando a identificar riscos e apontar condutas que favoreçam o cuidado seguro livre de danos preveníveis.

\section{- MÉTODO}

Este estudo constitui uma revisão integrativa realizada nos meses de janeiro e fevereiro de 2016, buscando responder a seguinte pergunta norteadora: O que há disponível na literatura científica atual sobre a atuação do enfermeiro na segurança do paciente cirúrgico?

Para tanto, foi realizada uma busca de artigos científicos nas bases de dados Scientific Electronic Library Online (SciELO), Base de Dados de Enfermagem (BDENF) e Literatura Latino-Americana e do Caribe em Ciências da Saúde (LILACS); através dos seguintes descritores: Segurança do Paciente (Patient Safety), Enfermagem Cirúrgica (Surgical Nursing), Centro Cirúrgico (Surgery Center), Enfermagem Perioperatória (Perioperative Nursing).

Tais descritores, antes do início da busca dos artigos, foram verificados na consulta aos Descritores em Ciências da Saúde (DeCS) e no Medical Subject Headings (MeSH) e foram cruzados utilizando o indicador AND da seguinte forma: Segurança do Paciente AND Enfermagem Cirúrgica, Segurança do Paciente AND Centro Cirúrgico, Segurança do Paciente AND Centro Cirúrgico AND Enfermagem Perioperatória e Segurança do Paciente AND Enfermagem Cirúrgica.

Os critérios de inclusão estabelecidos foram: artigos disponíveis na íntegra com um recorte temporal de 2011 a 2016, nos idiomas português, inglês e espanhol e que respondessem à pergunta norteadora do estudo. Foram excluídos livros, manuais, dissertações, teses, monografias e relatos de casos e experiência, bem como os artigos de revisão.

Os autores definiram as seguintes fases para a realização da revisão integrativa: elaboração da pergunta norteadora; busca/amostragem na literatura e coleta de dados (por três revisores independentes); análise crítica dos estudos incluídos; discussão dos resultados e apresentação da revisão integrativa ${ }^{(11)}$. Tais fases foram utilizadas nesta pesquisa visando alcançar o objetivo proposto.

\section{RESUltADOS}

Na busca inicial para a realização desta revisão integrativa, foram encontradas 280 publicações nas bases de dados LILACS, BDENF e SciELO, destas 252 foram excluídas por não abordarem a temática analisada. Assim, 28 publicações foram selecionadas para esta revisão, uma vez que atenderam aos critérios de inclusão preestabelecidos e trouxeram contribuições relevantes à discussão proposta pelo estudo.

Na Tabela 1, estão os resultados encontrados nas bases de dados LILACS, BDENF e SciELO, de acordo 
com o cruzamento dos descritores citados anteriormente.

Tabela 1 - Artigos encontrados e selecionados nas bases de dados LILACS, BDENF e SciELO sobre segurança do paciente cirúrgico na assistência de saúde, 2011 a 2016. Recife, PE, Brasil, 2016

\section{Descritores Segurança do paciente AND Enfermagem cirúrgica}

\begin{tabular}{lccc}
\hline Banco de dados & LILACS & BDENF & SciELO \\
\hline Artigos encontrados & 19 & 9 & 110 \\
\hline Artigos selecionados & 2 & 4 & 7 \\
\hline Descritores Segurança do paciente AND Centro cirúrgico & & & \\
\hline Banco de dados & LILACS & BDENF & SciELO \\
\hline Artigos encontrados & 19 & 15 & 68 \\
\hline Artigos selecionados & 6 & 4 & 8 \\
\hline Descritores Segurança do paciente AND Centro cirúrgico AND Enfermagem perioperatória & \\
\hline Banco de dados & LILACS & BDENF & SciELO \\
\hline Artigos encontrados & 3 & 1 & 25 \\
\hline Artigos selecionados & 2 & 1 & 10 \\
\hline Descritores Segurança do paciente AND Enfermagem cirúrgica AND Enfermagem pré-operatória & \\
\hline Banco de dados & LILACS & BDENF & SciELO \\
\hline Artigos encontrados & 4 & 4 & 5 \\
\hline Artigos selecionados & 1 & 2 & 5
\end{tabular}

Conforme o seguimento da análise dos artigos selecionados, foi possível destacar que a maior parte das publicações, nove (32,1\%), foi no ano de 2013, conforme distribuição exposta na Tabela 2.

Tabela 2 - Distribuição de artigos em número e percentual, conforme ano de publicação. Recife, PE, Brasil, 2016

\begin{tabular}{ccc} 
Ano de publicação & Quantidade de artigos & Percentual de artigos \% \\
\hline 2015 & 3 & 10,7 \\
\hline 2014 & 6 & 21,4 \\
\hline 2013 & 9 & 32,1 \\
\hline 2012 & 3 & 10,7 \\
\hline 2011 & 7 & 25,1
\end{tabular}

Em relação à formação dos autores, observou-se que 90,4\% (95), corresponde à área de enfermagem, seguida por nove $(8,5 \%)$ de medicina e um $(0,9 \%)$ de ciência aeroespacial.

A partir da pesquisa realizada, os artigos foram analisados e agrupados em categorias temáticas. A quantidade de artigos que fundamentaram cada categoria temática está descrita na Tabela 3.

Tabela 3 - Quantidade de artigos que fundamentaram cada categoria temática, conforme assuntos abordados. Recife, PE, Brasil, 2016

\section{Categoria}

Número de artigos selecionados

\begin{tabular}{lc}
\hline Reflexão e Avaliação acerca da assistência ofertada ao paciente cirúrgico & 10 \\
\hline Principais erros e fragilidades que põem em risco a segurança do paciente cirúrgico & 7 \\
\hline $\begin{array}{l}\text { Instrumentos e Estratégias para favorecer a qualidade da assistência e segurança do } \\
\text { paciente }\end{array}$ & 11 \\
\hline
\end{tabular}

Total de artigos selecionados

28 


\section{DISCUSSÃO}

Levando-se em consideração que a segurança do cliente está na redução e/ou atenuação de atos considerados inseguros no Centro Cirúrgico, bem como no emprego das melhores práticas, com o intuito de obter os resultados esperados, após a leitura completa dos 28 artigos foi possível identificar três categorias temáticas, conforme similaridade dos assuntos abordados em cada estudo: Reflexão e Avaliação acerca da assistência ofertada ao paciente cirúrgico, Principais erros e fragilidades que põem em risco a segurança do paciente cirúrgico, e Instrumentos e Estratégias para favorecer a qualidade da assistência e segurança do paciente.

\section{Reflexão e avaliação acerca da assistência ofertada ao paciente cirúrgico}

A assistência ao paciente cirúrgico, em qualquer uma das etapas operatórias, implica em uma série de ações que os profissionais devem atentar para manter a segurança do paciente. O cuidado no pré, trans e pós-operatório determina a qualidade da assistência prestada e a recuperação do cliente submetido ao procedimento cirúrgico. Estudos apontaram para a observação da etapa transoperatória no procedimento de preparo da sala cirúrgica, em que procedimentos como preparo seguro de medicamentos e hemoderivados e conferência de materiais e equipamentos cirúrgicos interferem diretamente na segurança do paciente ${ }^{(7)}$.

Além do transoperatório, na fase pós-operatória algumas atitudes de segurança podem ser implementadas para a assistência ao paciente; no caso da enfermagem, a aplicação de todas as etapas do processo de enfermagem é considerada uma metodologia eficaz para garantir não apenas a observação de sinais e sintomas à beira do leito, como também assistência integral e adequada até a completa reabilitação do paciente cirúrgico ${ }^{(12)}$.

Durante o pré-operatório, foram identificados fatores que interferem no enfrentamento positivo do paciente em relação ao procedimento cirúrgico, dentre eles destacamos a ansiedade. A ansiedade foi frequentemente associada ao procedimento cirúrgico em si, bem como à expectativa e preocupação com familiares. Nesse sentido, os profissionais podem utilizar recursos de tecnologia leve para trabalhar a ansiedade, como o encorajamento ao procedimento, o detalhamento das ações assistenciais a serem realizadas e o trabalho conjunto com os familiares reforçando a fé e a esperança ${ }^{(13)}$.

Ressalta-se neste contexto a importância do trabalho do enfermeiro na aproximação do paciente com sua espiritualidade, isentando-se de preferências pessoais por credos religiosos, compreendendo que esse dispositivo é essencial para que haja um melhor enfrentamento do procedimento cirúrgico pelo paciente, bem como um estreitamento da interface enfermeiro/paciente ${ }^{(13)}$, o que vai ao encontro do achado deste estudo, em que se percebe mais autores da área de enfermagem empenhados em discutir, através de publicações de estudos, práticas mais seguras em Centro Cirúrgico em prol de uma assistência de qualidade.

Nesse contexto, reforçar a cultura de segurança é essencial para garantir um ambiente adequado ao paciente, com riscos minimizados. Em avaliação da cultura de segurança, estudos apontaram para fragilidades na percepção dos profissionais em identificar um clima favorável à segurança do paciente no desenvolvimento de suas atividades no ambiente cirúrgico ${ }^{(10)}$.

Em relação à percepção do paciente acerca do cuidado perioperatório, evidenciou-se que a comunicação, o diálogo, as informações, o respeito e a orientação pré-operatória embasam a relação entre profissional de saúde e paciente. Muito embora tenham sido citados pelos pacientes como pontos favoráveis, são também percebidos como fragilidades, uma vez que a pouca importância dada pelos profissionais às orientações antes do procedimento cirúrgico, o diálogo, os atrasos e adiamentos não comunicados foram relacionados como dificultadores dessa relação ${ }^{(14)}$.

A comunicação entre os membros da equipe multidisciplinar de saúde também foi considerada como fator influenciador para segurança do paciente. Existindo comunicação ineficaz, os membros da equipe se põem em um ambiente favorável ao erro e à descontinuidade da assistência. A segurança do paciente não pode ser atribuída como responsabilidade de apenas uma categoria profissional, estando toda a equipe responsabilizada por preservar e promover essa segurança ${ }^{(15)}$. 


\section{Principais erros e fragilidades que põem em risco a segurança do paciente cirúrgico}

As falhas e os eventos adversos que acometem o paciente cirúrgico podem the trazer inúmeras consequências, inclusive com agravos permanentes. As falhas podem envolver recursos materiais, tecnológicos e humanos. Em se tratando de recursos humanos, um fator que influencia a segurança do paciente é a concentração no procedimento que está sendo realizado. Estudos apontaram que ocorrem, em média, 60 interrupções ou distrações durante o ato cirúrgico, na maioria das vezes desencadeadas pelo movimento de pessoas na sala. Em pacientes mais graves, submetidos ao controle de danos, a incidência de distrações foi ainda maior. A média de ruídos na sala de cirurgia foi muito elevada, com barulho próximo ao de um secador de cabelos ${ }^{(16)}$.

Tratando-se de ocorrência de eventos adversos, foi observada em um estudo a ocorrência de 42 eventos notificados. Especificamente em ocorrências relacionadas à administração de medicamentos, a prevalência de incidentes foi estimada em $48 \%$, tendo como fatores relacionados à sua ocorrência o tempo de internação igual ou superior a quatro dias, a prescrição de três ou mais medicamentos por dia e a realização de intervenção cirúrgica ${ }^{(17)}$.

No ambiente intraoperatório, as fragilidades que põem o paciente em risco são inúmeras, destacandose a ocorrência de lesões na pele durante o procedimento. Estudo observou a ocorrência de lesões na pele ao término do procedimento anestésico-cirúrgico em $74 \%$ dos pacientes. Nesses casos, destacou-se como fatores de risco para ocorrência desse tipo de lesões o não uso de recursos como protetores para manutenção corpórea, tempo cirúrgico prolongado e obesidade ou sobrepeso ${ }^{(18)}$.

Outra fragilidade, também evidenciada nessa pesquisa, foi em relação ao estresse entre os profissionais enfermeiros da unidade cirúrgica. Verificou-se em estudo que as atividades concebidas como administrativas e relacionadas à administração de pessoal foram consideradas mais estressantes. Nos resultados, 50\% das enfermeiras entrevistadas apresentavam estresse considerado de médio e alto e relataram a resolução de problemas como mecanismo para alívio deste problema ${ }^{(19)}$.

\section{Instrumentos e estratégias para favorecer a qualidade da assistência e segurança do paciente}

Diante de várias fragilidades e potenciais riscos encontrados nos estudos, buscou-se destacar também as possíveis estratégias e instrumentos utilizados para assegurar o paciente e garantir a qualidade da assistência prestada. A referência ao protocolo de cirurgia segura estabelecida pela Organização Mundial da Saúde (OMS) é frequente e relida por diversos autores. A utilização do sistema checklist apresenta ações específicas que permeiam três momentos do procedimento anestésico-cirúrgico (antes da indução anestésica, antes de iniciar a cirurgia e antes de o paciente sair da sala cirúrgica). A implementação do checklist confere o alcance do procedimento correto, pela equipe correta e paciente correto, tendo em vista estabelecer processos seguros na realização da cirurgia ${ }^{(1)}$.

Apesar da recomendação do uso desse protocolo pela OMS e grande parte das instituições hospitalares institucionalizarem a realização do checklist, a adesão efetiva dessa prática ainda está aquém do esperado para influenciar na segurança do paciente. Estudos verificaram que não houve adesão significativa na realização do checklist na conferência das informações de jejum, tricotomia, ausência de esmaltes e adornos, identificação do paciente e do sítio operatório, disponibilidade de sangue, verificação da lateralidade, apresentação da equipe e contagem de materiais na sala ${ }^{(20-21)}$.

Apesar da não adesão, após a utilização as equipes avaliaram positivamente os benefícios agregados com a conferência do protocolo, indicando maior segurança durante a realização do procedimento. Contudo, os participantes do estudo não referiram perceber melhorias na comunicação interpessoal na equipe multidisciplinar de saúde, mesmo com o uso do checklist ${ }^{(22)}$.

Outro mecanismo que podemos citar como benéfico para a minimização dos possíveis eventos adversos com o paciente cirúrgico é a comunicação efetiva. Tratando-se da enfermagem, percebeu-se que, quando esta se fez presente com informações e cuidados, os clientes relataram alto nível de satisfação e menor nível de ansiedade relacionada ao procedimento cirúrgico. Por outro lado, quando o profissional enfermeiro se distancia do paciente ou não presta as informações adequadas, são comumente referidos sentimentos de medo, insegurança e ansiedade ${ }^{(23)}$. 
Dessa forma, faz-se necessário enfatizar cada vez mais o caráter assistencial e clínico do enfermeiro, para que esse profissional possa executar atividades próximas ao paciente, reconhecendo assim as necessidades pertinentes a ele e podendo promover um cuidado individualizado e integral ao paciente cirúrgico, o que interfere também no reconhecimento e na valorização da profissão ${ }^{(23)}$.

O acesso a informações técnicas e atualizadas sobre assistência ao cliente cirúrgico na etapa pós-operatória também foi avaliado como valioso instrumento que influencia positivamente na segurança do paciente. Em estudo que avaliou a satisfação de profissionais enfermeiros sobre informações disponibilizadas em website sobre assistência de enfermagem pós-anestésica, esse meio de divulgação teve 99,67\% de aprovação dos usuários, sendo mais uma forma de acesso aos conteúdos para favorecer a prática mais segura aos pacientes no pós-anestésico ${ }^{(24)}$.

É possível ressaltar como limitação deste estudo a abrangência dos estudos analisados, percebendo-se a importância de uma reflexão mais aprofundada para que haja o conhecimento de variadas realidades, podendo-se assim fundamentar uma análise mais substanciada.

\section{CONSIDERAÇÕES FINAIS}

Ao final do estudo, percebemos que ainda há muito a percorrer nos caminhos da segurança efetiva para o paciente no período perioperatório, uma vez que encontramos muitos entraves que, de certa forma, desfavorecem a segurança deste, dentre os quais se destacam: os erros de montagem de sala cirúrgica; a não implementação de todas as etapas do processo de enfermagem; a ausência de diálogo entre o paciente e a equipe a respeito dos medos, da ansiedade, das dúvidas do pós-operatório; a falta de entendimento entre a equipe multiprofissional; a movimentação e os ruídos excessivos na sala cirúrgica; e a não realização do checklist recomendado pela OMS.

Conclui-se que as reflexões sobre a segurança do paciente e os processos do Centro Cirúrgico precisam ser aprofundadas, necessitando-se de novos estudos acerca do tema para novas discussões e busca de melhores práticas. É fundamental o engajamento de toda a equipe médica e de enfermagem para a assistência de qualidade e segura ao paciente cirúrgico, tentando-se eliminar os possíveis riscos e erros que ocorrem com frequência.

Seria oportuno entender a competência do enfermeiro do Centro Cirúrgico para além do fazer acontecer práticas organizacionais e voltar o olhar para a relação dessas práticas com a segurança do paciente.

No que concerne à limitação encontrada no desenvolvimento desta pesquisa, aponta-se para o pequeno quantitativo dos artigos selecionados, devido a não disponibilização na íntegra de alguns achados. Porém, mesmo assim, ao final deste estudo, com o alcance do objetivo estabelecido, identificaram-se as principais falhas e erros na atuação do enfermeiro na assistência ao paciente cirúrgico, deixando a desejar na segurança do paciente, bem como foram sugeridas soluções para melhoria da assistência.

Diante do exposto, espera-se que esta pesquisa traga contribuições para o aprimoramento e desenvolvimento do conhecimento científico sobre a atuação do enfermeiro na segurança do paciente.

Com isso, almeja-se que este estudo se torne uma ferramenta de divulgação desta temática, incentivando o desenvolvimento de outras pesquisas, tendo em vista não só a relevância científica que apresenta, mas também a relevância social diante da melhoria da assistência oferecida aos pacientes cirúrgicos.

\section{- REFERÊNCIAS}

1. Grigoleto ARL, Gimenes FRE, Avelar MCQ. Segurança do cliente e as ações frente ao procedimento cirúrgico. Rev. Eletr. Enf. [Internet] 2011; 13(2) [acesso em 24 fev 2016]. Disponível: http://dx.doi.org/10.5216/ree.v13i2.10326.

2. Secanell M, Orrego C, Vila M, Vallverdu H, Mora N, Oller A, et al. A surgical safety checklist implementation: experience of a start-up phase of a collaborative project in hospitals of Catalonia, Spain. Med Clin (Barc). 2014; 143(Suppl 1): $17-24$.

3. Zapata AIP, Samaniego MG, Cuéllar ER, Esteban EMA, Camara AG, López PR. Detection of adverse events in general 
surgery using the "Trigger Tool" methodology. Cir Esp. [Internet] 2015; 93(2) [acesso em 19 fev 2016]. Disponível: http:// dx.doi.org/10.1016/j.ciresp.2014.08.007.

4. Spruce L. Back to basics: implementing the surgical checklist. AORN J. 2014;100(5):465-73.

5. World Health Organization (WHO). Safe Surgery Saves Lives. [Internet] 2013 [acesso em 24 fev 2016]. Disponível: http://www.who.int/patientsafety/safesurgery/en/index.html.

6. Manrique BT, Soler LM, Bonmati AN, Montesinos MJL, Roche FP. Patient safety in the operating room and documentary quality related to infection and hospitalization. Acta paul. enferm. 2015; 28(4): 355-60.

7. Lima AM, Sousa CS, da Cunha ALSM. Segurança do paciente e montagem de sala operatória: estudo de reflexão. Rev enferm UFPE on line. 2013; 7(1): 289-94.

8. dos Santos J, Henckmeier L, Benedet SA. O impacto da orientação pré-operatória na recuperação do paciente cirúrgico. Enferm. Foco. 2011; 2(3): 184-7.

9. Conrardy JA, Brenek B, Myers S. Determining the state of knowledge for implementing the universal protocol recommendations: an integrative review of the literature. AORN J. 2010; 92(2): 194-207.

10. da Correggio TC, Amante LN, Barbosa SFF. Avaliação da cultura de segurança do paciente em Centro Cirúrgico. Rev. SOBECC. 2014; 19(2): 67-73.

11. de Souza MT, da Silva MD, de Carvalho R. Revisão Integrativa: o que é e como fazer. Eistein. [Internet] 2010; 8(1) [acesso em 26 fev 2016]. Disponível: http://apps.einstein.br/revista/arquivos/PDF/1134-Einsteinv8n1_p102-106_port.pdf.

12. Duarte SCM, Stipp MAC, Mesquita MGR, da Silva MM. O cuidado de enfermagem no pós-operatório de cirurgia cardíaca: um estudo de caso. Esc. Anna Nery. 2012; 16(4): 657-65.

13. Sampaio CEP, Ribeiro DA, Marta CB, Seabra Junior HC, Rose E, Francisco MTR. Determinant factors of the anxiety and mechanisms of coping on general surgical procedures. J. res.: fundam. care. online. 2013; 5(4): 547-55.

14. Callegaro GD, Baggio MA, do Nascimento KC, Erdmann AL. Cuidado perioperatório sob o olhar do cliente cirúrgico. Rev. Rene. 2010; 11(3): 132-42.

15. Bohomol E, Tartali JA. Eventos adversos em pacientes cirúrgicos: conhecimento dos profissionais de enfermagem. Acta paul. enferm. 2013; 26(4): 376-81.

16. Pereira BMT, Pereira AMT, Correia CS, Marttos Jr. AC, Fiorelli RKA, Fraga GP. Interrupções e distrações na sala de cirurgia do trauma: entendendo a ameaça do erro humano. Rev. Col. Bras. Cir. 2011; 38(5): 292-8.

17. Paranaguá TTB, Bezerra ALQ, de Camargo e Silva AEB, de Azevedo Filho FM. Prevalência de incidentes sem dano e eventos adversos em uma clínica cirúrgica. Acta paul. enferm. 2013; 26(3): 256-62.

18. Barbosa MH, Oliva AMB, de Sousa Neto AL. Ocorrência de lesões perioperatórias por posicionamento cirúrgico. Rev Cubana Enfermer. 2011; 27(1): 31-41.

19. Guido LA, da Silva RM, Goulart CT, Kleinübing RE, Umann J. Estresse e coping entre enfermeiros de unidade cirúrgica de hospital universitário. Rev. Rene. 2012; 13(2): 428-36.

20. Maziero ECS, de Camargo e Silva AEB, Mantovani MF, Cruz EDA. Adesão ao uso de um checklist cirúrgico para segurança do paciente. Rev. Gaúcha Enferm. 2015; 36(4): 14-20.

21. Elias ACGP, Schmidt DRC, Yonekura CSI, Dias AO, Ursi ES, Silva RPJ, Feijo VBER. Avaliação da adesão ao checklist de cirurgia segura em hospital universitário público. Rev. Sobecc. 2015; 20(3): 128-33.

22. Pancieri AP, Santos BP, de Avila MAG, Braga EM. Safe surgery checklist: analysis of the safety and communication of teams from a teaching hospital. Rev. Gaúcha Enferm. 2013; 34(1): 71-8.

23. Razera APB, Braga EM. A importância da comunicação durante o período de recuperação pós-operatória. Rev. esc. enferm. USP. 2011; 45(3): 632-7.

24. Lins TH, Marin HF. Avaliação de website sobre assistência de enfermagem na sala de recuperação pós-anestésica. 
Acta paul. enferm. 2012; 25(1): 109-15. 